\title{
Estimation of Photon Flux of the Oxygen Lyman- $\alpha$ Line Emitted from the W7-X Plasmas
}

\author{
T. Fornal ${ }^{a, *}$, I. KSIĄżEK ${ }^{b}$, M. KuBKOWSKA ${ }^{a}$, \\ R. BURHENN ${ }^{c}$ AND W7-X TEAM ${ }^{c}$ \\ ${ }^{a}$ Institute of Plasma Physics and Laser Microfusion, Hery 23, 01-497 Warsaw, Poland \\ ${ }^{b}$ Institute of Physics, Opole University, Oleska 48, 45-052 Opole, Poland \\ ${ }^{c}$ Max-Planck-Institut für Plasmaphysik, Wendelsteinstr. 1, 17491 Greifswald, Germany \\ Doi: 10.12693/APhysPolA.138.638 \\ *e-mail: tomasz.fornal@ifpilm.pl
}

\begin{abstract}
The low- $Z$ impurities of the magnetic confined fusion plasmas can provide important information about the wall condition and plasma-wall interactions. In order to accomplish this aim, a special spectrometer called "C/O Monitor" was designed for the W7-X experiment. This system is dedicated to measure Lyman- $\alpha$ transitions of four low- $Z$ impurities: carbon $(3.4 \mathrm{~nm})$, oxygen $(1.9 \mathrm{~nm})$, nitrogen $(2.5 \mathrm{~nm})$ and boron $(4.9 \mathrm{~nm})$. It is a high throughput and high time resolution spectrometer which allows to measure the line intensities evolution of indicated elements including information of the background (continuum). The designed spectrometer consists of two vacuum chambers positioned nearly horizontally - each chamber containing two dispersive elements. Their lines of sight cross at the main magnetic axis of the W7-X plasma. The spectrometer's installation is planned prior to the next Operational Phase (OP 2) which is to be performed in 2021. The "C/O Monitor" system has entered the executive stage. In the "C/O Monitor" diagnostic system, it is important to determine the incoming photon flux and to estimate the detector's response and the topmost time resolution. Moreover, this might be relevant for a quantitative interpretation of the experimental data as well as for an attempt at the calibration of this system. As a consequence, the incoming photon flux from the Lyman- $\alpha$ transitions of selected ions should be analyzed. In this paper, the estimation of the photon flux of the oxygen Lyman- $\alpha$ line and the whole methodology are described.
\end{abstract}

topics: EUV/soft X-ray spectroscopy, plasma impurities, stellarator plasma, C/O Monitor, W7-X

\section{Introduction}

The measurement of the plasma content in the magnetically confined fusion experiments is a very essential task. It delivers some important information about the plasma composition and the concentration of the intrinsic impurities that are constantly present in the plasma and have strong impact on its power balance. Although the radiated power strongly depends on the amount of heavy impurity ions through which a large part of energy can be emitted, the usually higher concentration of low- $Z$ materials (originating from the interaction with shields for the plasma facing components or remnants of water layers in the plasma vessel) plays a significant role during plasma startup when temperature is still not high enough to fully ionize these species. Nevertheless, when the plasma temperature stabilizes at the intended level, the energies corresponding to the Lyman- $\alpha$ transitions of low- $Z$ elements are emitted mostly from the plasma boundary and monitoring of their content plays an important role from the operational and safety points of view.
There are many impurity diagnostic systems working at the Wendelstein 7-X (W7-X) stellarator [1] (which is currently one of the largest fusion devices of this type in the world), delivering information about the plasma composition. Some of them are dedicated to measure heavy elements [2-4] in the soft X-ray region, while others are dedicated to investigate VUV wavelength range for observation of light elements or lower ionization states of heavy impurities [5, 6]. The "C/O Monitor" is a new diagnostic system dedicated to examine the low- $Z$ impurities like $\mathrm{B}(\mathrm{V})$ $(4.9 \mathrm{~nm}), \mathrm{C}(\mathrm{VI})(3.4 \mathrm{~nm}), \mathrm{N}(\mathrm{VII})(2.5 \mathrm{~nm})$ and $\mathrm{O}$ (VIII) $(1.9 \mathrm{~nm})$. Observation of those ions will be essential for the long pulse operation of the machine and its safety.

Since the heat-loaded parts of the inner wall of the W7-X plasma vessel as well as the divertor are covered with graphite tiles, a sudden increase of the carbon line may be an indicator of a local overheating of the surface. Therefore, in order to avoid the damage of the inner plasma vessel components, it is necessary to monitor its level continuously. When the machine is vented during 
maintenance, the air is absorbed by the inner wall of the vessel. Before the restart of the machine, oxygen is partially removed by simultaneous baking and pumping as far as possible. During the operation, the remaining oxygen is then slowly released and its content constantly decreases shot-by-shot. Therefore, its monitoring will deliver information about the general wall conditioning.

In order to reduce the influence of higher and medium- $Z$ impurities (sputtering and outgassing from the walls) on the plasma parameters, the plasma vessel can be covered by a thin layer of low- $Z$ material. In the case of $\mathrm{W} 7-\mathrm{X}$ stellarator, it is boron whose monitoring will deliver information about the quality of the boron coating.

An enhanced level of nitrogen (besides some specific experiments when it is injected into the plasma) may be an indication of a possible leakage or malfunction of the vacuum section.

The main role of the " $\mathrm{C} / \mathrm{O}$ Monitor" is to monitor the emission of the selected ion species which can also be done with the use of classical emission spectroscopy. For the electron temperatures achieved in W7-X, these impurity species (B, C, $\mathrm{N}$, and $\mathrm{O}$ ) are fully ionized in the plasma centre. The highest spectroscopically assessable ionization states are the H-like atoms, which for those elements are emitted in the far extreme ultraviolet (EUV) and soft X-ray range at the plasma boundary. Therefore, a spectroscopic system dedicated to study Lyman- $\alpha$ transitions of hydrogen-like atoms ought to be applied.

Since the "C/O Monitor" is designed to deliver fast, high quality information only about the evolution of the measured spectral line intensities, it will be a high throughput and high time resolution system (1 ms or better) but with a low spectral resolution. Consequently, the line shapes are not going to be investigated - only the line intensities.

\section{Geometrical assumption of the "C/O monitor" system}

The "C/O Monitor" is designed as a crystal spectrometer in Johann-geometry with cylindrically curved dispersive elements [7, 8]. In contrast to a monochromator, a polychromator reflects not only the central wavelength of the spectral line of interest but also its far wings i.e., the background level which has to be corrected in order to obtain the pure line intensity. Constant monitoring of spectral lines and the associated background will deliver continuous information about the behaviour of the specific plasma impurity which is being measured.

The system is constructed of two separated subspectrometer chambers positioned one over another, while the upper one is rotated upside down in relation to the bottom one (see Fig. 1). Each chamber contains two energy channels - one is dedicated to observation of $\mathrm{C}$ and $\mathrm{O}$ lines, while the second one is responsible for delivering information about

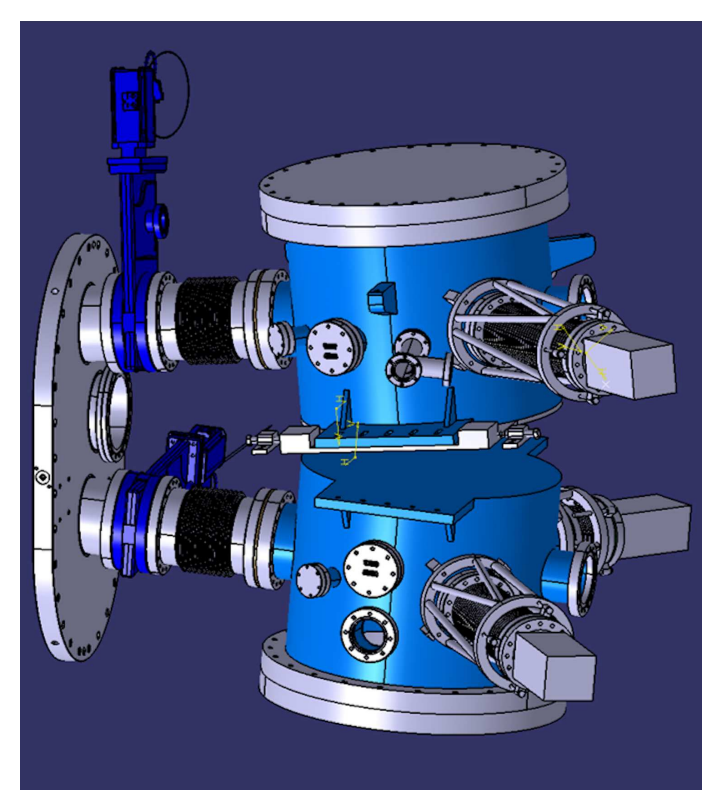

Fig. 1. Sub-spectrometer chambers of the "C/O Monitor" system.

the elements like B and N (see Fig. 1). It will be placed in a nearly horizontal position at the AEK 30 port of the W7-X stellarator. The lines of sight (LOS) of both sub-spectrometers are fixed and cross at the main magnetic axis.

The vacuum chambers are equipped with dispersive elements dedicated to reflect specific radiation energies. Since the reflectivity of applied dispersive elements strongly depends on the accuracy of their angular positions, precise rotational piezoelectrical drives for their fine tuning and grid collimators [9] are required. Finally, in order to limit or block the incoming light, apertures controlled by linear piezoelectrical drives have to be used. Since the incoming photon flux has been vertically collimated, the radiation corresponding to the specific spectral line is reflected to the output arm with the detector. For the initial phase, CCD cameras have been chosen due to their commercial availability and high sensitivity in the spectral range of interest reaching up to $90 \%$ [10]. Nevertheless, for the future operation other systems like micro channel plates (MCP), gas electron multiplier (GEM) or multistrip gaseous chamber (MSGC) are also considered [11].

Since for the plasma heating, an electron cyclotron resonance heating $(\mathrm{ECRH})$ system is used (together with a neutral beam injection and ion cyclotron resonance heating), it is necessary to prevent a reflected microwave stray radiation from reaching the interior of the spectroscopic system. Such a situation would result in an uncontrolled heating of the internal components leading to the system's damaging. For that reason, a special mesh for shielding the high frequency microwave radiation is used in the mechanical connection between sub-spectrometer chambers and the observation port at $\mathrm{W} 7-\mathrm{X}$. 
In order to estimate the detectors' performance in terms of the future "C/O Monitor" system operation, it was necessary to calculate the intensity of the photon flux reaching the detectors active areas. Therefore in the further section of this article, the methodology of the estimation of the calculated incoming photon flux for the Lyman- $\alpha$ oxygen line is presented, considering the geometrical setup and indicated influence of the specific subcomponents on the final results.

\section{Methodology of the photon flux estimation/calculations}

First of all, in order to estimate the photon flux it was necessary to determine the plasma volume observed by the designed diagnostic. Based on the computer-aided design (CAD) model of the system attached to the $\mathrm{W} 7-\mathrm{X}$ port, the maximum plasma volume seen by the single " $\mathrm{C} / \mathrm{O}$ monitor" channel has been determined to be approximately $10^{-2} \mathrm{~m}^{3}$. Since the CAD model is created, this volume was thoroughly meshed with spacing between points of $1 \mathrm{~cm}$ in all directions (see Fig. 2).

With the obtained matrix of points in a threedimensional volume $(x, y$, and $z$ in the Cartesian coordinate system of $\mathrm{W} 7-\mathrm{X})$, the next step was to choose the reference magnetic configuration of the stellarator. For each point of the considered volume and magnetic field configuration, the determination of the effective radius $R_{\text {eff }}$ [12] of the plasma volume has been performed. For this purpose, the variational moments equilibrium code (VMEC) was used which is dedicated to solve the magnetohydrodynamic force balance equations in a threedimensional space. Subsequently, for each obtained $R_{\text {eff }}$ point, the calculated profiles of $T_{e}\left(R_{\text {eff }}\right)$ and $n_{e}\left(R_{\text {eff }}\right)$ for a selected plasma heating scenario (which is just an example) were chosen [13] (see Fig. 3).

On the basis of information about the electron temperature distribution in the considered plasma volume, it was possible to calculate the appropriate local fractional abundance (FA) values for the specific H-like ions under the simplification of corona equilibrium. Together with the local temperaturedependent photon emission coefficient of the

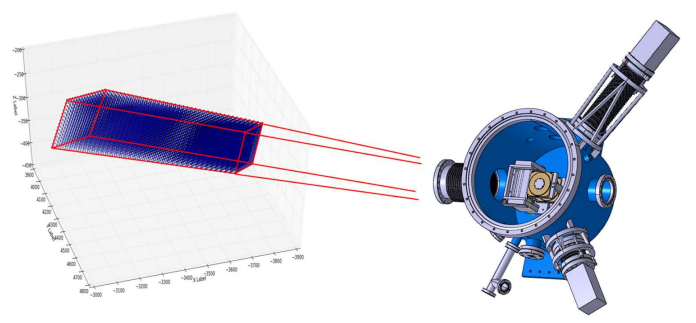

Fig. 2. The meshed plasma volume observed by the "C/O Monitor" system.

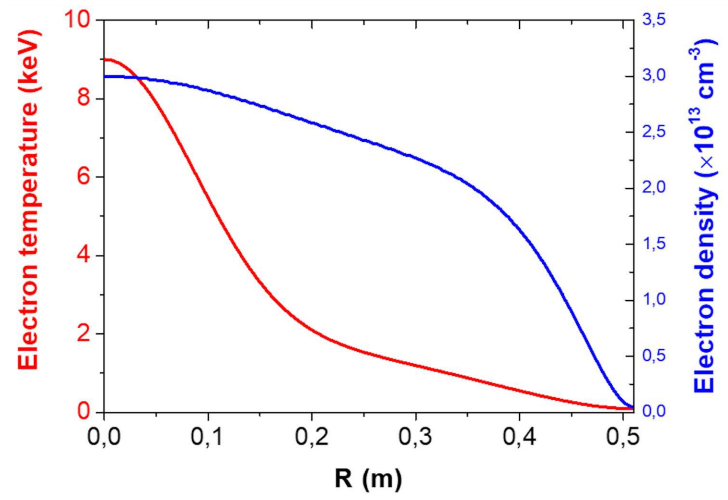

Fig. 3. Calculated electron density and temperature profiles of a selected plasma heating scenario planned for the future W7-X operation [13].

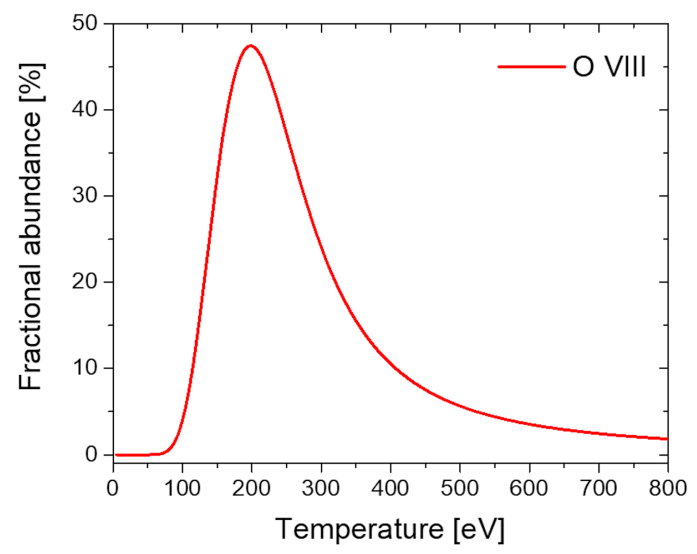

Fig. 4. Fractional abundance of the H-like oxygen density.

Lyman- $\alpha$ transitions and the electron density the corresponding local spectral emission can be obtained by [14]:

$$
C_{w}=\frac{n_{\mathrm{O}}}{n_{e}} \int \mathrm{PEC}^{\mathrm{O}}(R) n_{e}^{2}(R) \mathrm{FA}^{\mathrm{O}}(R) \mathrm{d} R,
$$

where $n_{\mathrm{O}}$ and $n_{e}\left[\mathrm{~cm}^{-3}\right]$ are the oxygen and electron densities, respectively constant with $R$. The photon emissivity coefficient is denoted as $\mathrm{PEC}^{\mathrm{O}}[\mathrm{ph} \mathrm{cm} / \mathrm{s}]$ and $\mathrm{FA}^{\mathrm{O}}$ reads as the fractional abundance of $\mathrm{O}$ (VIII) ions [15].

In the case of Lyman- $\alpha$ transition of $\mathrm{O}$ (VIII), the highest emission probability is for lower plasma temperatures in the range of $100-400 \mathrm{eV}$ with the maximum in $\approx 200 \mathrm{eV}$ (see Fig. 4). The obtained matrix of calculated points of $R_{\text {eff }}$ corresponding to the specific $n_{e}$ and $T_{e}$ as well as PEC values [15] and fractional abundances serve as an input to a dedicated software which has been created for quick numerical calculation of the estimation of line integrated emission. Finally, the emissivity radiated from each considered plasma volume $\left(R_{\text {eff }}\right.$ integrated) was calculated and the photon profile on $R_{\text {eff }}$ was determined. For this purpose, the whole input was used to make the estimation of the final line integrated intensity according to (1). 


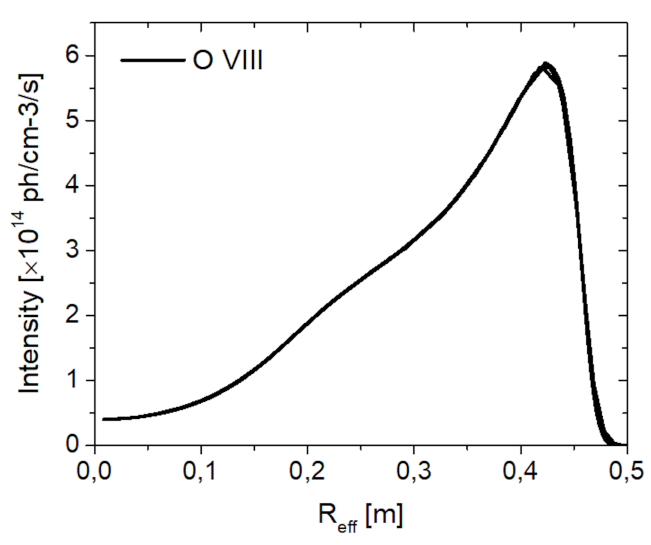

Fig. 5. Local O(VIII) emissivity in the function of $R_{\text {eff }}$.

This simplified methodology assumes that the local radiation is isotropic. The plasma opacity and impurity transport were not taken into account for this calculation. In the next section, the interpretation of the obtained results is given.

\section{Results}

The calculations show that the highest $\mathrm{O}$ (VIII) ion concentration is observed at the plasma boundary where the temperature is still low enough not to efficiently ionize to the fully stripped state (see Fig. 5).

The results were transformed into the total photon flux reaching the detector with reference to the maximum throughput that will be possible to process by the chosen detector model. The calculated number of photons emitted by the observed plasma volume in a $1 \mathrm{~s}$ time frame was at the level of $2.4 \times 10^{18}$.

In fact, the fraction emitted in the direction of the spectrometer (within its solid angle) was $\approx 1.6 \%$. The opacity of the plasma and inhomogeneous radial distribution of the impurity under study (transport processes) were neglected. However, this value was respectively reduced by the application of the grid collimator $(\approx 50 \%)$, ECRH shield $(\approx 50 \%)$ and dispersive elements reflectivity $(\approx 11 \%)$. Finally, in order to estimate the maximum performance of the detector with the requested high frequency of $1 \mathrm{~ms}$, the total photon flux reaching the detector active area was estimated to approximately $6.7 \times 10^{8} \mathrm{ph} / \mathrm{s}$. Since the dynamic range of the selected detector is 83000:1 (the ratio of bit depth of digitalisation), such intense radiation should be easily processed by the selected CCD camera model at the assumed detector operating frequency.

\section{Summary}

In this paper, the methodology of the photon flux estimation of Lyman- $\alpha$ transition is presented. The investigation with an example of oxygen shows that the highest photon flux of hydrogen-like atoms of oxygen is distributed mostly at the plasma boundary since the highest fractional abundance factor for this transition is achieved in the lower plasma temperatures (100-400 eV).

The main purpose of this work was to estimate (determine the order of magnitude) the photon flux expected at the focal plane of the spectrometer designed for W7-X. The results obtained on the level $6.7 \times 10^{8} \mathrm{ph} / \mathrm{s}$ confirmed that the total photon influx is at the sufficient level. The CCD cameras selected for the first operation phase of the "C/O Monitor" system can be the right choice for this purpose.

\section{Acknowledgments}

This work has been carried out within the framework of the EUROfusion Consortium and has received funding from the Euroatom research and training programme 2014-2018 and 2019-2020 under grant agreement No. 633053. The views and opinions expressed herein do not necessarily reflect those of the European Commission. This scientific work has partly been supported by the Polish Ministry of Science and Higher Education within the framework of the scientific financial resources for the years 2014-2020 allocated for the realization of the international co-financed project.

\section{References}

[1] R.C. Wolf, A. Ali, A. Alonso et al., Nucl. Fusion 57, 102020 (2017).

[2] A. Langenberg, N.A. Pablant, T. Wegner et al. Rev. Sci. Instrum. 89, $10 \mathrm{G} 101$ (2018).

[3] M. Kubkowska, A. Czarnecka, T. Fornal et al., Rev. Sci. Instrum. 89, 10F111 (2018).

[4] M. Kubkowska, A. Czarnecka, T. Fornal et al., Fusion Eng. Des. 136, 58 (2018).

[5] B. Buttenschön, R. Burhenn, M. Kubkowska et al., in: Proc. 43 rd EPS Conf. on Controlled Fusion Plasma Physics, 2016, Vol. 40A, 2016, P4.012.

[6] M. Kubkowska, B. Buttenschön, A. Langenberg et al., Probl. At. Sci. Technol. Ser. Plasma Phys. 118, 312 (2018).

[7] P. Beiersdorfer, S. Von Goeler, M. Bitter, K.W. Hill, R.A. Hulse, R.S. Walling, Rev. Sci. Instrum. 60, 895 (1989).

[8] R. Barnsley, N.J. Peacock, J. Dunn, I.M. Melnick, I.H. Coffey, J.A. Rainnie, M.R. Tarbutt, N. Nelms, Cit. Rev. Sci. Instrum. 74, 2388 (2003).

[9] D.L. McKenzie, P.B. Landecker, J.H. Underwood, Sci. Res. Counc. Symp. Tech. Sol. Cosm. X-Ray Spectrosc., Dorking 1975, p. 23. 
[10] Andor - Oxford Instruments.

[11] T. Fornal, I. Ksiazek, M. Kubkowska, J. Kaczmarczyk et al., Fusion Eng. Des. $B$ 146, 1681 (2019).

[12] B.Ph. van Milligen, A.L. Fraguas, J. Geiger, "Suggestions for Minor Radius Definitions in Stellarators".

[13] Y. Turkin, C.D. Beidler, H. Maaßberg, S. Murakami, V. Tribaldos, A. Wakasa, Phys. Plasmas 18, 022505 (2011).

[14] T. Nakano, A.E. Shumack, C.F. Maggi et al., in: 41st EPS Conf. Plasma Physics, EPS 2014.

[15] OPEN ADAS - Atomic Data and Analysis Structure, University of Strathclyde. 